\title{
Derivative Dispersion Relations
}

\author{
Regina Fonseca Ávila*† \\ Instituto de Matemática, Estatística e Computação Científica \\ Universidade Estadual de Campinas, UNICAMP \\ 13083-970 Campinas, SP, Brazil \\ E-mail: rfalifi.unicamp.br \\ Márcio José Menon \\ Instituto de Física Gleb Wataghin \\ Universidade Estadual de Campinas, UNICAMP \\ 13083-970 Campinas, SP, Brazil \\ E-mail: menon@ifi.unicamp.br
}

\begin{abstract}
We discuss some analytical and numerical aspects related to the replacement of integral dispersion relations by derivative relations and also the practical applicability of the derivative approach in the investigation of high-energy elastic hadron-hadron scattering. Making use of a Monopole Pomeron model and singly subtracted integral and derivative dispersion relations, we present the results of fits to the experimental data on the total cross sections and the ratio of the real to the imaginary part of the forward elastic scattering amplitude (proton-proton and antiproton-proton interactions). The emphasis is on the region of low energies and, in particular, we show that once the subtraction constant is used as a free fit parameter the derivative approach is equivalent to the integral approach even below the energy cutoff of the fitted data.
\end{abstract}

Fourth International Winter Conference on Mathematical Methods in Physics

09 - 13 August 2004

Centro Brasileiro de Pesquisas Fisicas (CBPF/MCT), Rio de Janeiro, Brazil

\footnotetext{
*Speaker.

${ }^{\dagger}$ Fellow from FAPESP.
} 


\section{Introduction}

Dispersion relations constitute a traditional and important mathematical method in several areas of physics, not only as a formal theoretical result, as a powerful phenomenological framework [1]. The practical applicability of the dispersion relations in high-energy particle interactions has received a renewed interest, mainly due to the lack of a pure fi eld theoretical description of the strong (hadronic) interactions in the sector named soft diffractive scattering (small momentum transfer and therefore large distances), which includes diffraction dissociation and elastic scattering. In fact, despite the success of the Quantum Chromodynamics (QCD) in the treatment of hard and semi-hard scattering (large and medium momentum transfer, respectively), the increase of the coupling constant when going to the soft sector does not allow the use of perturbative techniques and presently it is not know how to obtain the soft scattering states, directly, from a pure nonperturbative QCD approach.

At this stage general principles and theorems of the underlying fi eld theory, as unitarity, analyticity and crossing, constitute a formal framework for phenomenological developments aimed to work as a bridge between microscopic concepts (quarks and gluons) and the experimental data. In this context dispersion relations, as consequence of the principles of analyticity and crossing, play an important role, since they provide connections between the real and imaginary parts of the elastic scattering amplitude as function of the energy. Originally they have been introduced in the form of integral dispersion relations (IDR) [1, 2]. However, despite the important results that have been obtained, two limitations characterize the applicability of the integral forms, namely their non-local character (in order to obtain the real part of the amplitude the imaginary part must be known for all values of the energy) and the restricted classes of functions that allow analytical integration.

These shortcomings have been avoided in the region of high energies (center-of-mass energy above $10 \mathrm{GeV}$ ) with the introduction of dispersion relations in the differential form [3], the derivative dispersion relations (DDR), which have provided new insights in the dispersion relation techniques. A recent review and critical analysis on the formal and practical aspects of the DDR, with references to outstanding results and works on the subject, may be found in [ $₫$ ] (see also [5] and [6]).

In this communication, we investigate the real and imaginary parts of the elastic scattering amplitude, from proton-proton $(p p)$ and antiproton-proton $(\bar{p} p)$ interactions, by means of both IDR and DDR with one subtraction. Making use of a Monopole Pomeron model we obtain good descriptions of the experimental data on the total cross sections and the ratio of the real to the imaginary part of the forward amplitude. Our focus is the region of low energies and we show that, once the subtraction constant is used as a free fi t parameter, the derivative approach is equivalent to the integral approach even below the energy cutoff of the fi tted data.

The manuscript is organized as follows. In Sec. 2 we recall the physical quantities associated with the hadronic elastic scattering to be be investigated through the dispersion relations. In Sec. 3 we shortly review the main steps connecting IDR and DDR and in Sec. 4 we discuss the fi ts to the experimental data in the context of the Monopole Pomeron model. The results and conclusions are the contents of Sec. 5 . 


\section{Elastic Hadron Scattering}

For equal masses elastic scattering, the complex amplitude is expressed in terms of the Mandelstam variables, usually the center of mass energy squared $s$ and the square of the four-momentum transfer $t: F(s, t)=\operatorname{Re} F(s, t)+\mathrm{i} \operatorname{Im} F(s, t)$. In the forward direction $(t=0)$ the most important physical quantities that characterize the elastic scattering are the total cross section and the $\rho$ parameter. The former is given by the optical theorem, which at high energies reads

$$
\sigma_{\mathrm{tot}}(s)=\frac{\operatorname{Im} F(s, t=0)}{s},
$$

and the latter is defi ned by

$$
\rho(s)=\frac{\operatorname{Re} F(s, t=0)}{\operatorname{Im} F(s, t=0)} .
$$

Analyticity and Crossing connect the scattering amplitudes for particle-particle and particleantiparticle reactions, which are treated as crossed channels. From the experimental point of view the $p p$ and $\bar{p} p$ scattering correspond to the highest energy interval with available data and for that reason our analysis will be based on these reactions. In that case the above principles state that the corresponding amplitudes are the boundary value of an analytic function of $s$ and $t$ taken as complex variables. The amplitudes for these channels are expressed in terms of crossing even $(+)$ and odd (-) functions of the kinematical variables:

$$
F_{p p}=\frac{F_{+}+F_{-}}{2}, \quad F_{-p p}=\frac{F_{+}-F_{-}}{2} .
$$

Since $\sigma_{\text {tot }}$ and $\rho(s)$ are expressed in terms of the real and imaginary parts of the scattering amplitude they constitute useful and natural quantities to be investigate by means of dispersion relations, expressed in terms of crossing even and odd amplitudes, and that is the point we are interested in.

\section{Derivative Dispersion Relations}

The experimental information on the increase of the total cross sections suggest the use of IDR with one subtraction [2]. At high energies and with poles removed, they are expressed by

$$
\begin{gathered}
\operatorname{Re} F_{+}(s)=K+\frac{2 s^{2}}{\pi} P \int_{s_{0}}^{+\infty} \mathrm{d} s^{\prime} \frac{1}{s^{\prime}\left(s^{\prime 2}-s^{2}\right)} \operatorname{Im} F_{+}\left(s^{\prime}\right), \\
\operatorname{Re} F_{-}(s)=\frac{2 s}{\pi} P \int_{s_{0}}^{+\infty} \mathrm{d} s^{\prime} \frac{1}{\left(s^{\prime 2}-s^{2}\right)} \operatorname{Im} F_{-}\left(s^{\prime}\right),
\end{gathered}
$$

where $K$ is the subtraction constant, and for $p p$ and $\bar{p} p$ scattering, $s_{0}=2 m^{2} \sim 1.8 \mathrm{GeV}^{2}$.

The detailed replacement of the above formulas by the derivative forms may be found in [ 4 ]. In what follows we only recall the essential steps and the approximations involved. For the even amplitude we defi ne $s^{\prime}=\mathrm{e}^{\xi^{\prime}}, s=\mathrm{e}^{\xi}$ and $g\left(\xi^{\prime}\right)=\operatorname{Im} F_{+}\left(\mathrm{e}^{\xi^{\prime}}\right) / \mathrm{e}^{\xi^{\prime}}$ so that 


$$
\operatorname{Re} F_{+}\left(\mathrm{e}^{\xi}\right)-K=\frac{2 \mathrm{e}^{2 \xi}}{\pi} P \int_{\ln s_{0}}^{+\infty} \frac{g\left(\xi^{\prime}\right) \mathrm{e}^{\xi^{\prime}}}{\mathrm{e}^{2 \xi^{\prime}}-\mathrm{e}^{2 \xi}} \mathrm{d} \xi^{\prime}=\frac{\mathrm{e}^{\xi}}{\pi} P \int_{\ln s_{0}}^{+\infty} \frac{g\left(\xi^{\prime}\right)}{\sinh \left(\xi^{\prime}-\xi\right)} \mathrm{d} \xi^{\prime} .
$$

For the class of functions that are entire in the logarithm of $s$, we perform the Taylor expansion of the function $g\left(\xi^{\prime}\right)$ and then integrate term by term. At the high energy limit, we consider the approximation $s_{0}=2 m^{2} \rightarrow 0$, which allows to express

$$
\operatorname{Re} F_{+}\left(\mathrm{e}^{\xi}\right)-K=\frac{\mathrm{e}^{\xi}}{\pi} \sum_{n=0}^{\infty} \frac{g^{(n)}(\xi)}{n !} P \int_{-\infty}^{+\infty} \frac{\left(\xi^{\prime}-\xi\right)^{n}}{\sinh \left(\xi^{\prime}-\xi\right)} \mathrm{d} \xi^{\prime}
$$

and, after some analytical steps [4], we arrive at the DDR for the even amplitude,

$$
\frac{\operatorname{Re} F_{+}(s)}{s}=\frac{K}{s}+\tan \left[\frac{\pi}{2} \frac{\mathrm{d}}{\mathrm{d} \ln s}\right] \frac{\operatorname{Im} F_{+}(s)}{s} .
$$

With analogous procedure for the odd amplitude we obtain:

$$
\frac{\operatorname{Re} F_{-}(s)}{s}=\tan \left[\frac{\pi}{2}\left(1+\frac{\mathrm{d}}{\mathrm{d} \ln s}\right)\right] \frac{\operatorname{Im} F_{-}(s)}{s} .
$$

These are the DDR for entire functions in $\ln s$ and intended for the high-energy region.

\section{Fits with the Monopole Pomeron Model}

In order to test the applicability of the DDR and compare the results with those obtained with the IDR, we shall consider as input a well known parametrization for the total cross section and investigate the results for the $\rho$ parameter, obtained through simultaneous fi ts of both quantities to the experimental data on $p p$ and $\bar{p} p$ scattering. In the extended Monopole Pomeron model the total cross sections for these reactions are given by

$$
\sigma_{\mathrm{tot}}(s)=X s^{\varepsilon}+Y_{+} s^{-\eta_{+}}+\tau Y_{-} s^{-\eta_{-}},
$$

where the first term represents the exchange of a single Pomeron, the other two the secondary Reggeons and $\tau=+1(-1)$ for $p p(\bar{p} p)$ amplitudes. The couplings $X, Y_{+}, Y_{-}$and the powers $\varepsilon$, $\eta_{+}, \eta_{-}$(associated with the Pomeron and Reggeons intercepts) are free fi t parameters.

The point is to analytically evaluate $\rho(s)$ in Eq. (2.2) from the above parametrization and the procedure is as follows. From Eq. (4.1) we determine the imaginary parts of the amplitudes $F_{p p}$ and $F_{\bar{p} p}$ by means of the optical theorem (2.1) and then the imaginary parts of the crossing even and odd amplitudes by inverting Eq. (2.3). These, in turn, are used as inputs in the IDR (3.1), (3.2) or in the DDR (3.3), (3.4), leading to the determination of the real parts of the amplitudes and, through (2.3), to $\rho(s)$ as given (2.2).

As demonstrated in [П], with the above procedure, the results with the IDR read

$$
\begin{aligned}
\rho(s) & =\frac{1}{\sigma_{\text {tot }}(s)}\left\{\frac{K}{s} \pm Y_{-} s^{-\eta_{-}} \cot \left(\eta_{-} \frac{\pi}{2}\right)+X s^{\varepsilon} \tan \left(\varepsilon \frac{\pi}{2}\right)-Y_{+} s^{-\eta_{+}} \tan \left(\eta_{+} \frac{\pi}{2}\right)\right. \\
& \left.+\frac{2}{\pi} \sum_{j=0}^{\infty}\left( \pm \frac{Y_{-} s_{0}{ }^{1-\eta_{-}}}{s\left(2 j+2-\eta_{-}\right)}+\frac{X s_{0} \varepsilon}{2 j+1+\varepsilon}+\frac{Y_{+} s_{0}-\eta_{+}}{2 j+1-\eta_{+}}\right)\left(\frac{s_{0}}{s}\right)^{2 j+1}\right\}
\end{aligned}
$$




\begin{tabular}{|c|cc|cc|}
\hline & \multicolumn{2}{|c|}{ Integral Dispersion Relations } & \multicolumn{2}{c|}{ Derivative Disperion Relations } \\
& $K=0$ & $K$ free & $K=0$ & $K$ free \\
\hline$\varepsilon$ & $0.0874 \pm 0.0024$ & $0.0895 \pm 0.0025$ & $0.0814 \pm 0.0021$ & $0.0895 \pm 0.0025$ \\
$\eta_{+}$ & $0.399 \pm 0.014$ & $0.382 \pm 0.015$ & $0.450 \pm 0.012$ & $0.382 \pm 0.015$ \\
$\eta_{-}$ & $0.533 \pm 0.012$ & $0.545 \pm 0.013$ & $0.573 \pm 0.015$ & $0.545 \pm 0.013$ \\
$X(\mathrm{mb})$ & $20.12 \pm 0.51$ & $19.61 \pm 0.53$ & $21.44 \pm 0.44$ & $19.61 \pm 0.53$ \\
$Y_{+}(\mathrm{mb})$ & $68.9 \pm 2.1$ & $66.4 \pm 2.0$ & $78.0 \pm 2.0$ & $66.4 \pm 2.0$ \\
$Y_{-}(\mathrm{mb})$ & $-32.4 \pm 1.8$ & $-33.8 \pm 1.9$ & $-38.8 \pm 2.6$ & $-33.8 \pm 1.9$ \\
$K$ & 0 & $-48 \pm 15$ & 0 & $69 \pm 18$ \\
$\chi^{2} / D O F$ & 1.14 & 1.10 & 1.64 & 1.10 \\
\hline
\end{tabular}

Table 1: Results of the simultaneous fits to $\sigma_{\text {tot }}$ and $\rho$ (238 data points) with the Monopole Pomeron Model, either with the IDR or DDR and $K=0$ or $K$ as a free fit parameter.

and those obtained with the DDR are given by

$$
\rho(s)=\frac{1}{\sigma_{\text {tot }}(s)}\left\{\frac{K}{s} \pm Y_{-} s^{-\eta_{-}} \cot \left(\eta_{-} \frac{\pi}{2}\right)+X s^{\varepsilon} \tan \left(\varepsilon \frac{\pi}{2}\right)-Y_{+} s^{-\eta_{+}} \tan \left(\eta_{+} \frac{\pi}{2}\right)\right\},
$$

where the signs \pm apply for $p p(+)$ and $\bar{p} p(-)$ scattering. We notice that the above analytical results, provided by the DDR, are the same as those obtained with the IDR in the high-energy limit, $s_{0}=2 m^{2} \rightarrow 0$.

The tests with the IDR or DDR have been made through fi ts of Eqs. (4.1) - (4.2) or (4.1) (4.3), respectively, to the experimental data on $\sigma_{\text {tot }}$ and $\rho$ from $p p$ and $\bar{p} p$ scattering. We have used the experimental data compiled and analyzed by the Particle Data Group [7], with the statistical and systematic errors added in quadrature and energy above $\sqrt{s}_{\min }=5 \mathrm{GeV}$ ( 238 data points). The fi ts have been performed with the program CERN Minuit and the errors in the fit parameters correspond to an increase of the $\chi^{2}$ by one unit.

In order to investigate the effect of the subtraction constant we have made fi ts taking $K=0$ or using $K$ as a free fi t parameter. Summarizing, we have four variants: IDR or DDR and in each case $K=0$ or $K$ as a free fi t parameter.

\section{Results and Conclusions}

The numerical results and statistical information from all the cases analyzed are displayed in Table 1. The comparison of the fi $t$ results with the experimental data are shown in Fig. $1(K=0)$ and in Fig. 2 ( $K$ as a free fi t parameter), where we have also included the data below the energy cutoff for the fit $(2<\sqrt{s}<5 \mathrm{GeV})$.

From Table 1, the best statistical results were obtained with $K$ as a free fi t parameter $\left(\chi^{2} / D O F\right.$ $=1.10$ ). From Fig. 1 we see that for $K=0$, the differences between the results with IDR and with DDR are signifi cant at both low and high energies for $\rho(s)$ and at high energies for $\sigma_{\text {ot }}(s)$. On the other hand, from Fig. 2, in the case that $K$ is a free parameter, the results with the DDR and with the IDR are coincident. That can also be seen from Table 1 , since the values of the fit parameters 

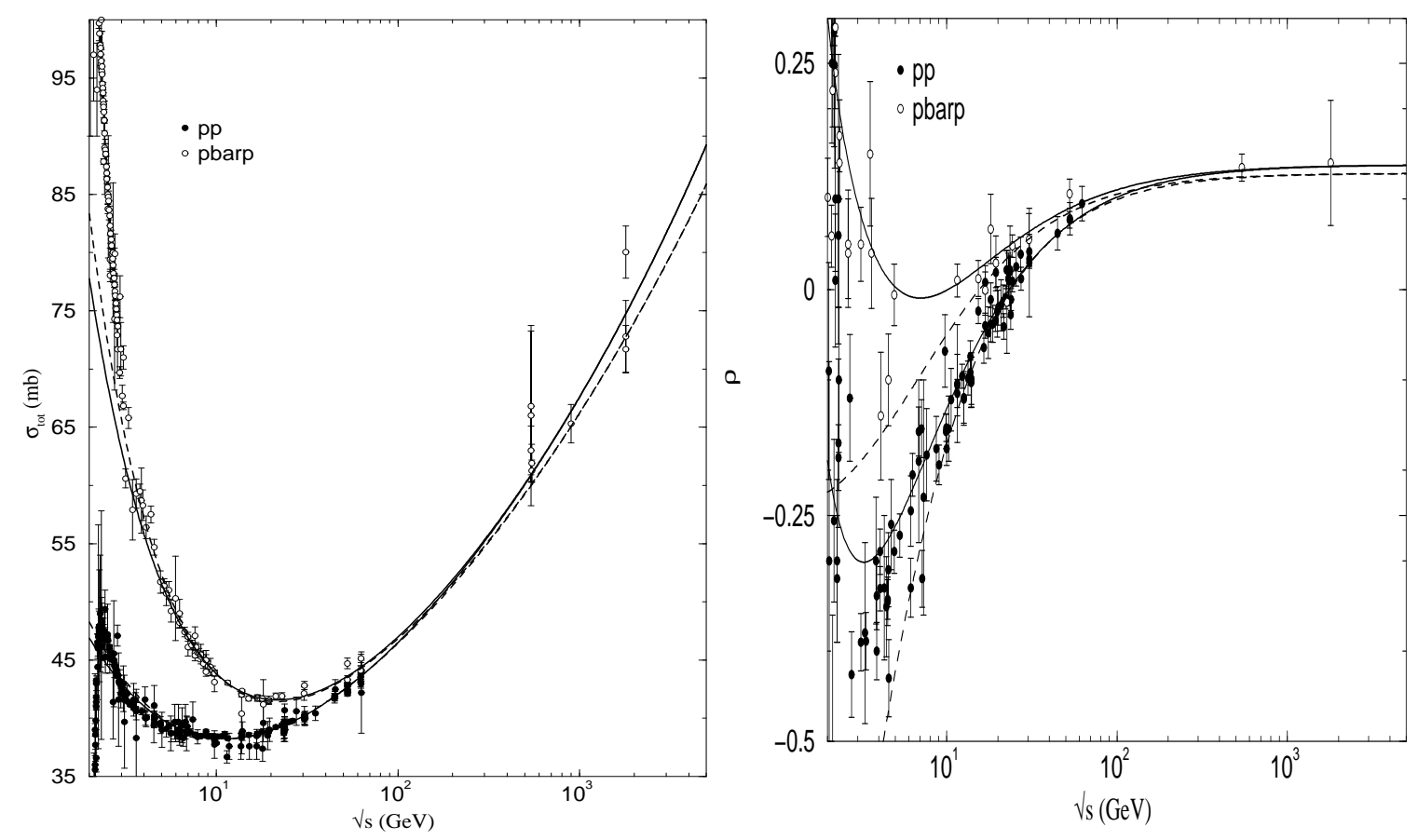

Figure 1: Simultaneous fit of the Monopole Pomeron model to $\sigma_{\text {tot }}$ and $\rho(\sqrt{s}>5 \mathrm{GeV})$, assuming $K=0$ and using either the IDR (solid) or the DDR (dashed).
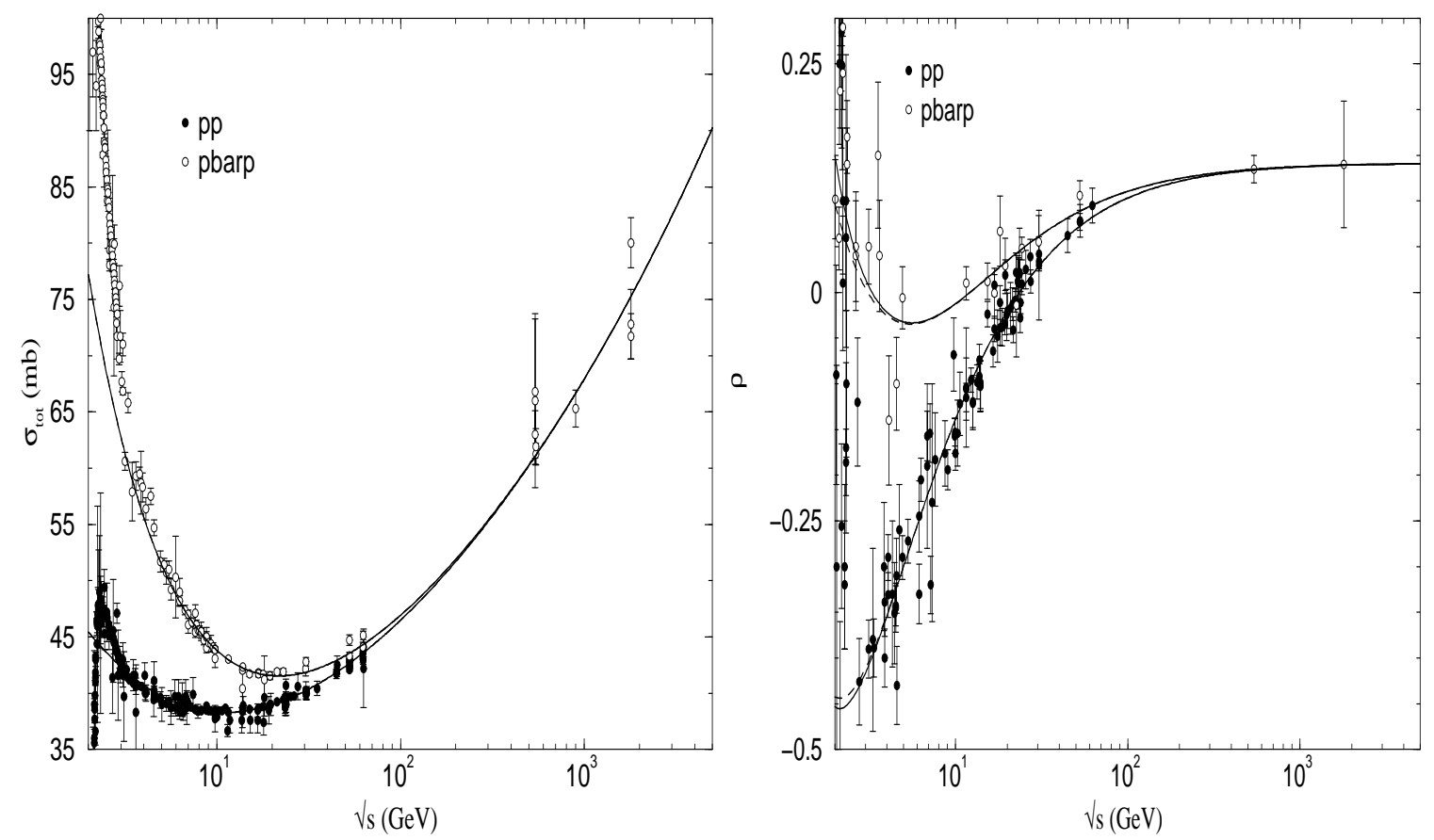

Figure 2: Simultaneous fit of the Monopole Pomeron model to $\sigma_{\text {tot }}$ and $\rho(\sqrt{s}>5 \mathrm{GeV})$, with $K$ as a free fit parameter and using either the IDR (solid) or the DDR (dashed). 
(and the $\chi^{2} / D O F$ ) are the same up to 3 signifi cant fi gures. Moreover, in this case, the description of the experimental data taking part in the fi $\mathrm{t}$ (above $5 \mathrm{GeV}$ ) is quite good (see also [\$]).

As we have discussed, the DDR bring enclosed the high-energy approximation $\left(s_{0}=2 m^{2} \rightarrow 0\right.$ from the IDR). Despite of this, even the data below the energy cutoff for the fit $(5 \mathrm{GeV})$ is well described in the case that $K$ is a fi t parameter. The analytical explanation for this effect may be found in Eq. (4.2): the leading contribution in the series expansion depends on $1 / s$ and, therefore, can be absorbed by the term with the subtraction constant, since it also depends on $1 / s$. We note that the subtraction constant is a consequence of the polynomial bound on the scattering amplitude and therefore has a well founded mathematical bases. All that shows the important role played by the subtraction constant in the practical equivalence of the integral and derivative approaches. We conclude that $K$ can not be disregarded or assumed as zero in any reliable use of the derivative dispersion relations.

This work was based on the Monopole Pomeron model for the forward scattering amplitude and singly subtracted dispersion relations. Further tests with dipole and tripole contributions, as well as twice subtracted dispersion relations, may bring new insights on the subject.

\section{References}

[1] H. Burkhardt, Dispersion relation dynamics, North-Holland, Amsterdam, 1969; H.M. Nussenzveig, Causality and dispersion relations, Academic Press, New York 1972.

[2] M.L. Goldberger, Y. Nambu, R. Oehme, Dispersion relations for nucleon-nucleon scattering, Ann. Phys. 2 (1957) 226; P. Söding, Real part of the proton-proton and antiproton-proton forward scattering amplitude at high energies, Phys. Lett. 8 (1964) 285.

[3] V.N. Gribov, A.A. Migdal, Properties of the Pomeranchuk pole and the branch cuts related to it at low momentum transfers, Sov. J. Nucl. Phys. 8 (1969) 583; J.B. Bronzan, G.L. Kane, U.P. Sukhatme, Obtaining real parts of the scattering amplitudes directly from cross section data using derivative analyticity relations, Phys. Lett. B 49 (1974) 272; K. Kang, B. Nicolescu, Models for hadron-hadron scattering at high energies and rising total cross sections, Phys. Rev. D 11 (1975) 2461.

[4] R.F. Ávila, M.J. Menon, Critical analysis of derivative dispersion relations at high energies, Nucl. Phys. A 744 (2004) 249 [hep-ph / 0309028$].$

[5] P. Kolár, J. Fischer, Dispersion relations in differential form, in Proceedings Blois Workshop on Elastic and Diffractive Scattering, Prague, 2001, IOP, 305 [hep-th/0110233]; On the validity and practical applicability of derivative analyticity relations, J. Math. Phys. 25 (1984) 2538.

[6] M.J. Menon, A.E. Motter, B.M. Pimentel, Differential dispersion relations with an arbitrary number of subtractions: a recursive approach, Phys. Lett. B 451 (1999) 207 [hep-th/9810196]; R.F. Ávila, E.G.S. Luna, M.J. Menon, Analytical models and forward scattering from accelerator to cosmic-ray energies, Phys. Rev. D 67 (2003) 054020 [hep-ph/0212234].

[7] Particle Data Group, K. Hagiwara et al. Phys. Rev. D 66 (2002) 010001, the full data sets are available at http://pdg.lbl.gov.

[8] E. Martinov, J.R. Cudell, O.V. Selyugin, Integral and derivative dispersion relations, analysis of the forward scattering data, Eur. Phys. J. C 33 (2004) 533 [hep-ph/0311019]. 